\title{
Automated IR determination of petroleum products in water based on sequential injection analysis
}

\author{
Marina Falkova*, Christina Vakh, Andrey Shishov, Ekaterina Zubakina, Aleksey Moskvin, \\ Leonid Moskvin, Andrey Bulatov \\ Department of Analytical Chemistry, St. Petersburg State University, RU-198504 Saint Petersburg, Russia
}

\section{A R T I C L E I N F O}

\section{Article history:}

Received 20 February 2015

Received in revised form

14 May 2015

Accepted 18 May 2015

Available online 21 May 2015

Keywords:

Petroleum products

Water

Extraction

Sequential injection analysis

Infrared detection

\begin{abstract}
A B S T R A C T
The simple and easy performed automated method for the IR determination of petroleum products (PP) in water using extraction-chromatographic cartridges has been developed. The method assumes two stages: on-site extraction of PP during a sampling by using extraction-chromatographic cartridges and subsequent determination of the extracted PP using sequential injection analysis (SIA) with IR detection. The appropriate experimental conditions for extraction of the dissolved in water PP and for automated SIA procedure were investigated. The calibration plot constructed using the developed procedure was linear in the range of 3-200 $\mu \mathrm{g} \mathrm{L}^{-1}$. The limit of detection (LOD), calculated from a blank test based on $3 \sigma$ was $1 \mu \mathrm{g} \mathrm{L}^{-1}$. The sample volume was $1 \mathrm{~L}$. The system throughput was found to be $12 \mathrm{~h}^{-1}$.
\end{abstract}

(c) 2015 Elsevier B.V. All rights reserved.

\section{Introduction}

Petroleum products inevitably are released into the environment and contaminate surface water bodies particularly near production or storage sites, but also escape accidentally during handling, transport or processing [1]. As it is well known [2,3], the petroleum products are very complex mixtures that contain primarily aliphatic and aromatic hydrocarbons and heterocycles. Currently, World Health Organization regulates the PP content (dissolved/emulsions) in water, which cannot exceed levels higher than $300 \mu \mathrm{g} \mathrm{L}^{-1}$ to provide a conservative level of protection [4]. Thus, one of the most important analytical tasks of environmental monitoring is the PP determination in the natural waters to assess levels of environmental pollution.

The four most commonly used PP testing methods include gas chromatography (GC) [5-10], infrared absorption (IR) [11], spectrofluorimetry (SFL) [12] and gravimetric analysis (GA) [13,14] (Table 1). GC-based methods detect a broad range of hydrocarbons, provide both sensitivity and selectivity, and can be used for petroleum hydrocarbon identification as well as quantification. However, there are difficulties in the automation of whole procedures and determination of total PP content in water. The main advantage of the IR method is the insignificant dependence of absorption on the type of petroleum hydrocarbons, mainly

\footnotetext{
*Corresponding author. Fax: + 79213316858.

E-mail address: albina_my_mail@mail.ru (M. Falkova).
}

containing in water samples. However, the IR method assumes the delivery of large volume of water sample in laboratory (up to $1 \mathrm{~L}$ per determination). The SFL is the most sensitive method for the determination of aromatic hydrocarbons and heterocycles, but the emission of hydrocarbons depends on their structure [15]. GA methods may be useful for water samples with a high PP concentration.

Generally GC, IR, SFL, and GA methods include conventional liquid-liquid extraction (LLE) of PP from the water samples to another water-immiscible solvent. Commonly used solvents for extraction are carbon tetrachloride, tetrachlorethylene, trifluorotrichloroethane and hexane. Although LLE is relatively simple and inexpensive, it has many drawbacks, among them the need to use large quantities of solvents. In order to achieve the desired enrichment factor, the excess solvent requires removal by evaporation, and extract cleanup may also be necessary. Also the solid-phase (SPME) [7], headspace solid-phase microextraction (HS-SPME) [8] and flow solid-phase (FSPME) [9] microextraction techniques are proposed to the hydrocarbons determination in water by GC. The SPME is based on the extraction of hydrocarbons from the water using a microsyringe equipped by poly(dimethylsiloxane) fiber in a needle. In the case of HS-SPME fiber with a poly(dimethylsiloxane) coating is placed in a headspace of a water sample. For the FSPME water is passed through a syringe steel needle filled with the Tenax GR sorbent at the rate of $2 \mathrm{~mL} \mathrm{~min}{ }^{-1}$. The double solid-phase extraction (SPE) is suggested for the determination of aliphatic and aromatic hydrocarbons in 


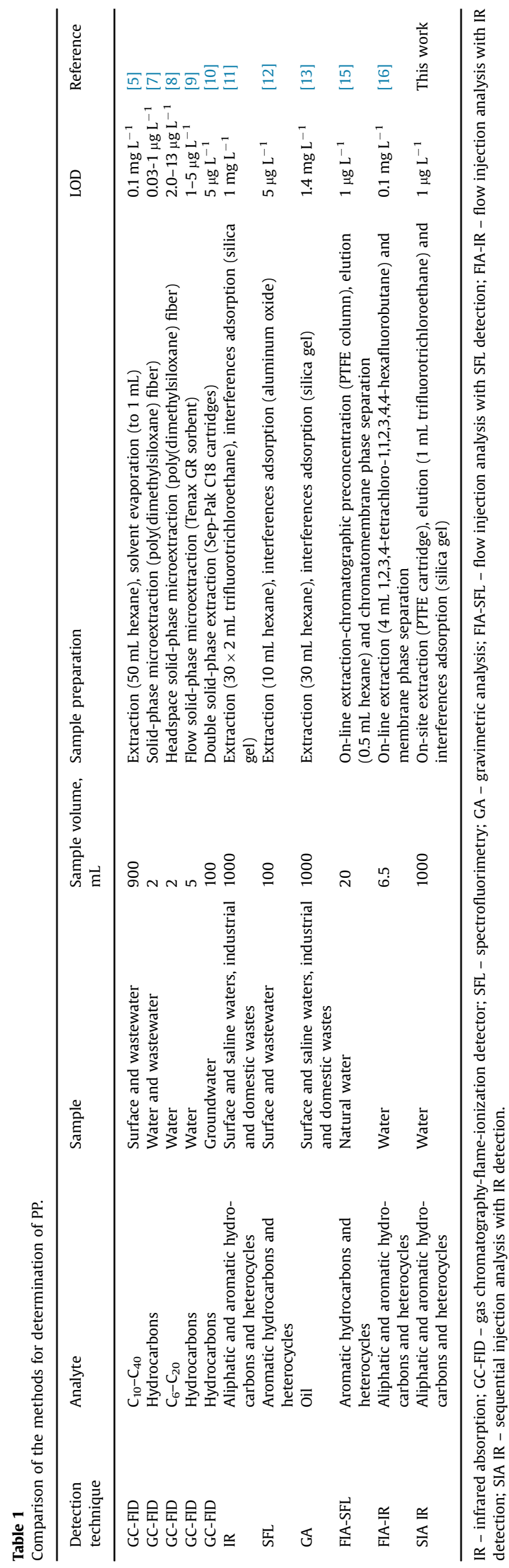

groundwater [10]. By using the first SPE (reverse phase), the hydrocarbons are extracted from groundwater sample, meanwhile the second SPE is accomplished for fractionating hydrocarbons into aliphatic and aromatic hydrocarbons. Finally SPME and SPE devices are introduced into the injection port of gas chromatograph to thermal desorption of hydrocarbons.

The important and rapidly growing trend in modern analytical chemistry is the automation of analysis. Currently, the automation of analytical procedures based on flow analysis is intensively developing. To the best of our knowledge, only two articles have been devoted to the PP determination in water based on flow system $[15,16]$.

The developed flow-injection method with IR detection assumes the mixing carbon tetrachloride with an aqueous carrier containing the sample into an extraction coil. Finally the two phases are separated into a membrane separator and the organic phase is transferred to the detection cell for absorbance measurement [16]. The main disadvantage of this flow method is low sensitivity $\left(0.1 \mathrm{mg} \mathrm{L}^{-1}\right)$ because it is impossible to increase water and organic segments volume ratio to PP preconcentration. In the case of FIA fluorimetric determination of aromatic hydrocarbons and heterocycles in water [15], the on-line preconcentration of analytes is carried out into the extraction-chromatographic column. The extract is eluted by hexane with the following separation of extract from aqueous phase in chromatomembrane cell and aromatic hydrocarbons and heterocycles are detected, wherein all saturated hydrocarbons are ignored. This fact leads to the essential underestimation of the results in the determination of PP by this detection method when the sample contents, for example, petrol, kerosene and other same fractions.

The aim of this work was to develop an automated method for IR determination of total PP content (aliphatic and aromatic hydrocarbons and heterocycles) in water. To increase the sensitivity and exclude the mentioned disadvantages of LLE the special extraction-chromatographic cartridges have been developed and used for on-site extraction of PP during a sampling.

\section{Experimental}

\subsection{Chemicals}

Analytical grade chemicals and distilled water were used throughout the experiments. Carbon tetrachloride, tetrachlorethylene, trifluorotrichloroethane, hexane, isooctane, hexadecane, benzene, isopropyl alcohol, aluminum oxide and silica gel for chromatography (01-0.25 mm) were purchased from Sigma-Aldrich. Different types of PP (gasoline, diesel, transformer, industrial and vaseline oils) were obtained from the co-operation partner (Gasprom, Russia).

The $0.5 \mathrm{~g} \mathrm{~L}^{-1}$ stock solutions of PP in a water-soluble matrix were prepared by dissolving PP in isopropyl alcohol. The working water solutions/emulsions of PP were prepared by dissolving the corresponding aliquots of $0.5 \mathrm{~g} \mathrm{~L}^{-1}$ stock solutions in water and adjusting the volume up to $1 \mathrm{~L}$ by adding distilled water, and then flask contents were carefully mixed and immediately analyzed.

The calibration solutions were prepared by mixing isooctane, hexadecane and benzene at the volume ratio of 1.5:1.5:1 (OCB standard solution) and dissolution of this mixture in trifluorotrichloroethane, and stored in a sealed container to avoid evaporative loss.

\subsection{Apparatus}

\subsubsection{Sampling and sample preparation setup}

Extraction-chromatographic cartridges (polytetrafluoroethylene (PTFE)) (height - $20 \mathrm{~mm}, 5 \mathrm{~mm}$ i.d.) using for PP extraction were filled 


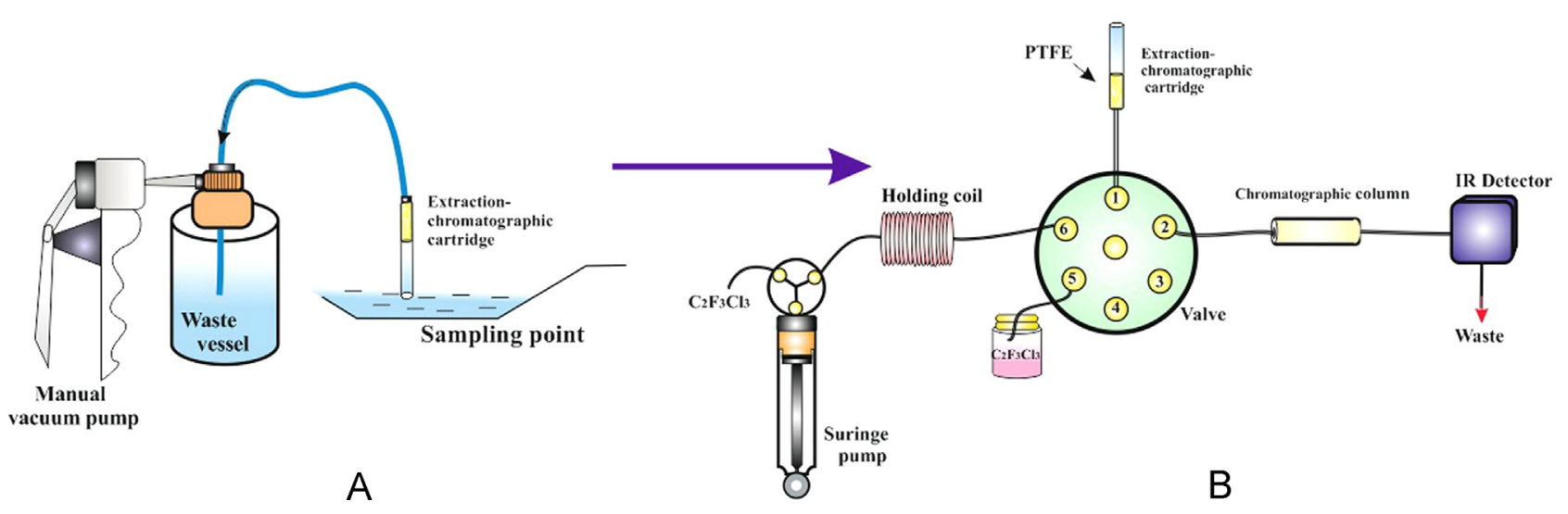

Fig. 1. (A) The scheme of sampling and exraction. (B) The SIA manifold for the IR determination of PP in water.

with block porous PTFE. The PTFE powder (Fluoroplast-4) was sintered at a temperature of $380^{\circ} \mathrm{C}$ for $5 \mathrm{~h}$ in metal form $\left(20 \times 50 \times 50 \mathrm{~mm}^{3}\right)$ to prepare a block porous PTFE. Then the PTFE block was crushed in a blender, and the fraction size of PTFE powder from 0.5 to $0.9 \mathrm{~mm}$ was selected. Cylindrical form (height $-10 \mathrm{~mm}$, diameter $-5 \mathrm{~mm}$ ) was filled with prepared fraction and PTFE powder was re-sintered at a temperature of $380{ }^{\circ} \mathrm{C}$ for $1.5 \mathrm{~h}$.

$0.5 \mathrm{~mL}$ of trifluorotrichloroethane was passed through the prepared cartridge for its activation. After that the cartridge was washed with $2 \mathrm{~mL}$ of the distilled water to remove the excess of trifluorotrichloroethane.

Aluminum oxide and silica gel chromatographic columns were used for the separation of polar components from extracts (height $-2 \mathrm{~mm}, 1.5 \mathrm{~mm}$ i.d.). Aluminum oxide was preliminary dried at $600{ }^{\circ} \mathrm{C}$ for $4 \mathrm{~h}$.

The manual vacuum pump with manometer "SM 16673» (Sartorius, Germany) was used for the sampling. The column was connected to the vacuum pump by using a silicone tube (Fig. 1A).

\subsubsection{Sequential injection setup}

The sequential injection manifold (Fig. 1B) for the determination of PP in water was based on a flow analyzer (Rosanalit, Russia). It included a syringe pump (SP) ensuring the reverse flow with $5 \mathrm{~mL}$ capacity, a 6-position solenoid valve (Cole-Parmer, Inc., USA), tubes for communications (PTFE, $0.5 \mathrm{~mm}$ of i.d.). The $2000 \mathrm{~cm}$ length holding coil (HC) from PTFE with $0.5 \mathrm{~mm}$ of i.d. was connected to the central port of the solenoid valve. The manifold was connected with an IR-spectrometer with a $40 \mathrm{~mm}$ path-length flow cell (Emi, Russia), an extraction-chromatographic cartridge and a silica gel chromatographic column (Section 2.2.1). Absorption registration was carried out in the $2700-3200 \mathrm{~cm}^{-1}$ spectral region. The analyzer was operated automatically by means of a computer.

\subsubsection{Batch experiments}

An RF-5301PC Spectrofluorophotometer (Shimadzu Scientific Instruments, Japan) was employed for batch spectrofluorimetric measurements.

\subsection{Sampling/extraction}

The extraction-chromatographic cartridge was immersed in water (depth $-0.5 \mathrm{~m}$ ), and $1 \mathrm{~L}$ of the water sample was directly pumped from the sampling place using a vacuum pump at the rate of $60 \mathrm{~mL} \mathrm{~min}{ }^{-1}$ through the extraction-chromatographic cartridge (Fig. 1A). After extraction the extraction-chromatographic cartridges could be stored for a day in a refrigerator.

\subsection{SIA operational protocol}

After on-site extraction of PP the extraction-chromatographic cartridge was included into the SIA manifold (valve position 1) (Fig. 1B). The procedure started with the aspiration of $1 \mathrm{~mL}$ of trifluorotrichloroethane from the reservoir flask (valve position 5) to the HC by movement of the SP. Trifluorotrichloroethane was then directed to the extraction-chromatographic cartridge (valve position 1) by the SP at the rate of $2 \mathrm{~mL} \mathrm{~min}^{-1}$. Afterwards, the obtained extract was aspirated to the HC and moderately pushed into the silica gel chromatographic column (valve position 2), where adsorption of polar components took place. After that, the PP extract was delivered from chromatographic column into the flow cell for IR detection, and the signal was measured under stopped-flow conditions for $20 \mathrm{~s}$. Subsequently, the solution was sent to the waste. The tubes and the flow cell were cleaned with trifluorotrichloroethane to prevent any cross-contamination.

\subsection{SFL operational protocol}

The $100 \mathrm{~mL}$ of $100 \mu \mathrm{g} \mathrm{L}^{-1}$ water solution of PP was placed into a separatory funnel and $10 \mathrm{~mL}$ of hexane was added. The mixture was shaken for $5 \mathrm{~min}$. After separation of the phases, the extract was separated, and then SFL determination of PP was performed at an excitation wavelength of $270 \mathrm{~nm}$ and fluorescence recording wavelength of $310 \mathrm{~nm}$ [12].

\section{Results and discussion}

\subsection{Preconcentration and SIA conditions}

For on-site preconcentration of PP from water the extractionchromatographic cartridge with immersed block PTFE was used. In the pores of block PTFE various solvents (carbon tetrachloride, tetrachlorethylene and trifluorotrichloroethane) were used as a stationary phase. Criterion for the choice of solvent was the absence of IR radiation absorption in the $2700-3200 \mathrm{~cm}^{-1}$ spectral region, where the absorption by PP, caused by the stretching vibrations of $\mathrm{CH}_{3}$ and $\mathrm{CH}_{2}$ groups of aliphatic, alicyclic compounds and side chains of aromatic hydrocarbons, as well as aromatic $\mathrm{CH}$-bonds, was observed. In addition, the solvent should be well fastened in the pores of block PTFE immersed in the extraction-chromatographic cartridge. Different volumes of $200 \mu \mathrm{g} \mathrm{L}^{-1}$ water solution of transformer oil were passed through the cartridge at the sampling rate under $50 \mathrm{~mL} \mathrm{~min}^{-1}$. It was found that by using carbon tetrachloride, tetrachlorethylene or trifluorotrichloroethane as the stationary phase, PP extraction efficiency has been determined to be equal to $95 \%$ by passing up to $10 \mathrm{~L}$ of PP water solution. In this case the 
concentration factor equal to $6 \times 10^{3}$ could be achieved, this fact allowed to significantly increase the sensitivity of PP detection. The removal of the organic phase from the block PTFE occurred, when the volume of passing aqueous sample was increased. For further studies trifluorotrichloroethane was chosen as a more environmentally friendly solvent.

The effect of the PTFE powder fractions size on the reproducibility of the analytical signal was investigated. The minimum RSD (1\%) was observed, using block PTFE, obtained from 0.5 to $0.9 \mathrm{~mm}$ PTFE fraction. By using the 1-2 mm PTFE fraction the stationary phase was removed during passing water and efficient extraction of PP did not occur. While using the 0.1-0.4 mm PTFE fraction the hydrodynamic resistance was increased and the RSD also increased to $7 \%$.

Furthermore the efficiency of PP extraction at various sampling rates of PP water solution was investigated. For this purpose, $1 \mathrm{~L}$ of $200 \mu \mathrm{g} \mathrm{L}^{-1}$ water solution of transformer oil was passed through the extraction-chromatographic cartridge. The univariate optimization method was applied, while the sampling rate was changed from 20 to $100 \mathrm{~mL} \mathrm{~min}^{-1}$. The results showed (Fig. 2A) that the satisfactory PP extraction was obtained at the sampling rate under $60 \mathrm{~mL} \mathrm{~min}^{-1}$, which was chosen for further experiments. At the next stage, the optimal volume of trifluorotrichloroethane required for the elution of PP under SIA conditions was chosen (Fig. 2B). Based on the obtained results, the optimal eluent volume was $1 \mathrm{~mL}$

It has been further found that filtration rate could be increased to $300 \mathrm{~mL} \mathrm{~min}^{-1}$ by three-fold increase of the cartridge size (height $-60 \mathrm{~mm}, 15 \mathrm{~mm}$ i.d.). However, $8 \mathrm{~mL}$ of the solvent was required for the elution of $\mathrm{PP}$; it was not justified due to the significant organic waste generation.

\subsection{Study of interferences}

The effect of different polar components encountered in water was investigated using model samples. Adequate recovery was taken to be an analytical response with a signal deviation up to $\pm 5 \%$. The results showed that the polar components such as glyceryl tristearate, phenol, alkylated phenols, organic acids (stearic, palmitic acid, etc.), naphthenic acids interfere at up to 2 -fold excess. Due to the polar components can interfere to the IR-determination of PP, the efficiency of their extraction was evaluated by moving of trifluorotrichloroethane through the aluminum oxide and silica gel columns. The effect of the polar components was investigated using the $100 \mathrm{mg} \mathrm{L}^{-1}$ solutions of mentioned polar components in trifluorotrichloroethane. The highest efficiency of polar components sorption was achieved by using silica gel as a sorbent, which was chosen for further studies. Therefore, it can be assumed that the developed procedure should be applicable for the determination of PP in water.

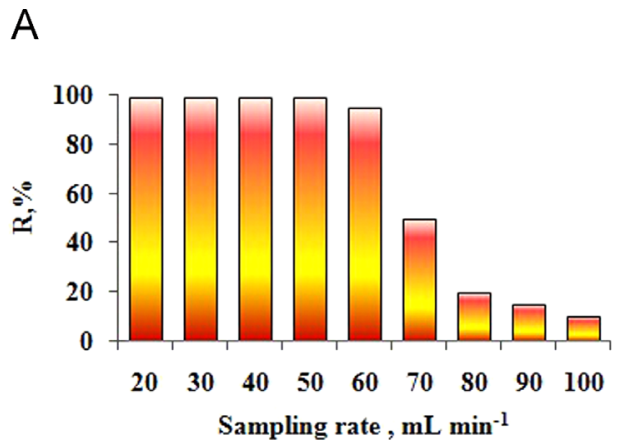

\subsection{Analytical performance}

The calibration plot constructed using OCB standard solutions in the SIA setup was linear in the range of $3-200 \mu \mathrm{g} \mathrm{L}^{-1}$ with correlation coefficients $\left(r^{2}\right)$ of 0.999 while the sample volume equals $1 \mathrm{~L}$. The limit of detection (LOD), calculated as three-times the standard deviation $(3 \mathrm{~s}$ ), and limit of quantification (LOQ), calculated as 10-times the standard deviation (10 s) of the blank test $(n=10)$, were assessed as $1 \mu \mathrm{g} \mathrm{L}^{-1}$ and $3 \mu \mathrm{g} \mathrm{L}^{-1}$, respectively. The suggested method showed satisfactory repeatability of the analytical response by evaluation of the relative standard deviation (RSD) from 10 replicate measurements of the PP in real samples, with a value from $1.4 \%$ to $4.6 \%$. The system throughput, assessed as the sampling frequency, was found to be $12 \mathrm{~h}^{-1}$.

\subsection{Analysis of model and real samples}

The developed method has been verified on the $100 \mu \mathrm{g} \mathrm{L}^{-1}$ water solutions of different types of PP. Moreover determination of PP in model solutions was performed by the SFL method, described in Section 2.5. In the case of IR detection (see Section 2.4.) added and found amounts of PP were almost identical (Fig. 3). A significant understating of results (Fig. 3) was observed in the determination of PP by the SFL method for gasoline and diesel samples, since they were mainly composed of aliphatic hydrocarbons, and the content of aromatic hydrocarbons and heterocycles was negligible in these PP.

The developed procedure was applied for the analysis of real samples: natural water from rivers and lakes. Samples were collected from 4 sample points in St. Petersburg (Russia). The water samples were directly pumped through the extraction-chromatographic cartridge using a vacuum pump at the rate of $60 \mathrm{~mL} \mathrm{~min}{ }^{-1}$. To carry out the recovery study, the water samples $(1 \mathrm{~L})$ were collected into the glass flasks by using bathometer and spiked with 0.5 and $1 \mathrm{~mL}$ of $10 \mathrm{mg} \mathrm{L}^{-1}$ solution of transformer oil in isopropyl alcohol and then flask contents were carefully mixed and immediately pumped through the extraction-chromatographic cartridges. After sampling the extraction-chromatographic cartridges were stored for a day in a refrigerator at the temperature $5{ }^{\circ} \mathrm{C}$. Analytical results measured by the proposed automated method are presented in Table 2 . The recoveries obtained for each of the water samples were within of $96.1-103.2 \%$ range which is considered to be accepfor this type of samples. The RSD does not exceed $5 \%$.

\section{Conclusion}

The new simple and easy performed automated method for the IR determination of total PP content in water has been developed. The developed procedure is based on the on-site extraction of PP

B

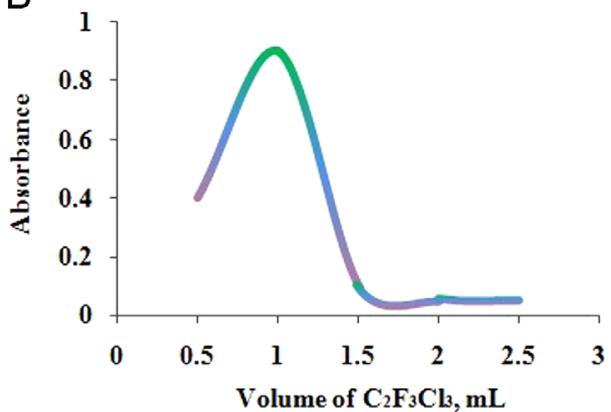

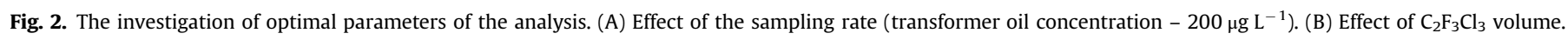






Fig. 3. The results of the PP determination in the model water solutions using IR and SFL detection $(n=3, P=0.95)$.

Table 2

The results of the PP determination in the natural waters $(n=3, P=0.95)$.

\begin{tabular}{lccl}
\hline Sample & Added, $\mu \mathrm{g} \mathrm{L}^{-1}$ & Found, $\mu \mathrm{g} \mathrm{L}^{-1}$ & Recovery \\
\hline River I & 0 & $111 \pm 10$ & - \\
& 5 & $115 \pm 8$ & 99.1 \\
\multirow{2}{*}{ River II } & 10 & $122 \pm 5$ & 100.8 \\
& 0 & $21 \pm 2$ & - \\
& 5 & $25 \pm 4$ & 96.1 \\
Lake I & 10 & $32 \pm 2$ & 103.2 \\
& 0 & $55 \pm 5$ & - \\
\multirow{2}{*}{ Lake II } & 5 & $60 \pm 4$ & 100.0 \\
& 10 & $64 \pm 5$ & 98.4 \\
& 0 & $73 \pm 6$ & - \\
& 5 & $79 \pm 8$ & 101.3 \\
\end{tabular}

during a sampling by using the extraction-chromatographic cartridges and subsequent sequential injection determination with IR detection. This method is more advantageous than the previously developed FIA methods because it is more sensitive and allows determining the total PP content (aliphatic and aromatic hydrocarbons and heterocycles).

\section{Novelty statement}

The first sequential injection IR method for the determination of total petroleum products (PP) in water has been presented. The extraction-chromatographic cartridges for the on-site sampling of PP have been proposed. New method is more advantageous than the previously developed flow methods because it is more sensitive and allows determining the total PP content (aliphatic and aromatic hydrocarbons and heterocycles).

\section{Acknowledgments}

Authors gratefully acknowledge the Russian Foundation for Basic Research for the financial support (Project no. 13-03-00031-a).

\section{References}

[1] S. Drozdova, W. Ritter, B. Lendl, E. Rosenberg, Fuel 113 (2013) 527-536.

[2] J.G. Speight, The Chemistry and Technology of Petroleum, fifth ed., Tailor and Francis, New York, 2014.

[3] K. Weggen, G. Pusch, H. Rischmüller, Oil and Gas in Ullmann's Encyclopedia of Industrial Chemistry, Wiley-VCH, Weinheim, 2005.

[4] WHO, Petroleum Products in Drinking-water, Background Document for Development of WHO Guidelines for Drinking-water Quality, WHO, Geneva, 2005.

[5] DIN ISO 9377-2:2000, Water quality - Part 2: method using solvent extraction and gas chromatography, International Organization for Standardisation, 2000.

[6] OSPAR Commission, Ref. no. 2005-15, OSPAR reference method of analysis for determination of the dispersed oil content in produced water, 2005.

[7] J.J. Langenfeld, S.B. Hawthorne, D.J. Miller, Anal. Chem. 68 (1996) 144-155.

[8] M. Tankiewicz, C. Morrison, M. Biziuk, Microchem. J. 108 (2013) 117-123.

[9] V.G. Berezkin, E.D. Makarov, B.V. Stolyarov, J. Chromatogr. A 985 (2003) 63-65.

[10] O.P. Jiméneza, R.M.P. Pastora, O.E. Segovia, S.R. Querencia, Talanta 131 (2015) 315-324.

[11] EPA Method 418.1, Total recoverable petroleum hydrocarbons by IR, Government Printing Office, Washington, DC, USA, 1978.

[12] Federal Environmental Regulations 14.1:2:4.128-98, Quantitative chemical analysis of water. Measurement of the concentration of petroleum products in natural, drinking, sewage water by fluorimetric method, 1998.

[13] EPA Method 413.1, Standard test method for oil and grease using gravimetric determination, 1978.

[14] EPA Method 1664, Revision A: $n$-hexane extracmaterial (HEM; oil and grease) and silica gel treated $n$-hexane extracmaterial (SGT-HEM: nonpolar material) by extraction and gravimetry, 1999.

[15] A.L. Moskvin, L.N. Moskvin, A.V. Moszhuchin, V.V. Fomin, Talanta 50 (1999) $113-120$.

[16] K. Hayakawa, Y. Yoneda, Y. Okamoto, T. Kumamaru, M. Ikeda, Anal. Sci. 15 (1999) 803-805. 